\title{
Regularidad superficial y adherencia en vías ciclistas - recomendaciones de diseño disponibles
}

\section{Surface regularity and skid resistance in cycle lane - Available Design Recommendations}

A. Barbudo ${ }^{(*)}$, J. R. Jiménez $^{(*)}$, E. F. Ledesma ${ }^{(*)}, \mathrm{M}^{\mathrm{a}}$. J. Sierra ${ }^{(* *)}$

\section{RESUMEN}

Este trabajo presenta una revisión de diferentes normativas y recomendaciones nacionales e internacionales de diseño de pavimentos de vías ciclistas y analiza las especificaciones en cuanto a regularidad superficial y resistencia al deslizamiento/ derrape contempladas en las mismas. Tras un estudio comparativo, se pone de manifiesto la carencia de criterios específicos de diseño y la necesidad de unificar métodos de ensayo normalizados y valores límites de aceptación y/o rechazo que garanticen la seguridad y comodidad de los nuevos tramos construidos, favoreciendo así la movilidad ciclista en las ciudades.

Palabras clave: Vías ciclistas; regularidad superficial; resistencia al deslizamiento; seguridad; pavimentos; capa de rodadura.

\begin{abstract}
This paper presents an overview of different national and international regulations and recommendations about pavement design in cycle lanes and analyzes the specifications for surface roughness and slip/skid resistance that each offers. After a comparative study, it highlights the lack of specific design criteria and the need to unify the standard test methods and limit values of acceptance and / or rejection to ensure the safety and comfort of the new constructed sections, thus promoting cycling mobility in cities.

Keywords: Cycle lanes; surface roughness; slip resistance; safety; surface paving; wearing course.

(*) Universidad de Córdoba (España).

${ }^{(* *)}$ Agencia de Obra Pública - Consejería de Fomento y Vivienda, Junta de Andalucía. Sevilla (España).

Persona de contacto/Corresponding author: jrjimenez@uco.es (J.R. Jiménez)
\end{abstract}

Cómo citar este artículo/Citation: Barbudo, A., Jiménez, J. R., Ledesma, E. F., Sierra, M ${ }^{\mathrm{a}}$. J. (2015). Regularidad superficial y adherencia en vías ciclistas - recomendaciones de diseño disponibles. Informes de la Construcción, 67(540): e124, doi: http://dx.doi. org/10.3989/ic.15.010.

Licencia / License: Salvo indicación contraria, todos los contenidos de la edición electrónica de Informes de la Construcción se distribuyen bajo una licencia de uso y distribución Creative Commons Reconocimiento no Comercial 3.o. España (cc-by-nc). 


\section{INTRODUCCIÓN}

La construcción de vías ciclistas representa una de las estrategias con mayor impacto dentro de los proyectos de transporte sostenible. Además de cumplir con las propias exigencias constructivas, las vías ciclistas deben otorgar condiciones de seguridad y comodidad a los usuarios, al objeto de mejorar su percepción ciudadana, aumentar el número de ciclistas urbanos e incrementar los viajes en bicicleta en detrimento de otros medios de transporte motorizados más contaminantes.

El firme constituye una de las partes más importantes en el diseño de vías ciclistas. Así, la elección del tipo de firme para una vía ciclista estará en función de una triple tipología de factores: consideraciones técnicas (capacidad de carga, regularidad superficial, resistencia al deslizamiento/derrape, drenaje y durabilidad), consideraciones económicas (costes de ejecución y mantenimiento), y adecuación al usuario e impacto (requerimientos/necesidades, integración y legibilidad) (1).

Un firme está compuesto por una serie de capas superpuestas con espesores diferentes en función de la capacidad portante de la explanada y del tipo de tráfico que soportan (2) (3). Así, el pavimento o capa de rodadura viene definida como la parte superior del firme que debe resistir los esfuerzos producidos por la circulación (3) (4), cuyas funciones son, por tanto, proveer una superficie de rodadura cómoda y segura, y proteger la capa base (5). En el caso de las vías ciclistas la capacidad de carga no viene determinada por el uso ciclista, sino que más importantes son las cargas sufridas durante la ejecución, el mantenimiento o en algún caso esporádico el uso de vehículos de emergencia (6) (7).

Por otro lado, la adherencia entre la rueda de la bicicleta y el pavimento es una de las características superficiales que tiene más influencia en la seguridad del ciclista, ya que permite acelerar, frenar y mantener en todo momento la trayectoria de la bicicleta sin riesgo de deslizamiento o derrape (2). Esta propiedad se mide mediante la resistencia al deslizamiento/ derrape y está íntimamente ligada con la textura superficial del pavimento (2).
En relación a la textura superficial hay que diferenciar entre la macrotextura o rugosidad y la microtextura o aspereza. La macrotextura depende de la granulometría y tamaño máximo del árido de la mezcla bituminosa, en carreteras está muy relacionada con la resistencia al deslizamiento del vehículo a velocidades superiores a $60 \mathrm{~km} / \mathrm{h}$ y pavimento mojado. La microtextura o aspereza depende de la textura superficial de los áridos, y la de los morteros en las mezclas bituminosas, e influyen mucho en la adherencia rueda-pavimento (8). Lo más adecuado es tener una microtextura áspera y una macrotextura rugosa, aunque esta última aumenta la resistencia a la rodadura y por lo tanto, el esfuerzo en la conducción. Por otro lado, la megatextura está relacionada con la puesta en obra o con diferentes fallos, como son los baches, el tipo de material también puede afectar a la megatextura, como por ejemplo los adoquines, baldosas, etc. (8), por lo que no afecta a la adherencia rueda-pavimento.

La macrotextura se mide a través del ensayo del círculo de arena (UNE-EN 13036-1:2010) o con texturómetros láser (UNEEN ISO 13473-1:2006). La microtextura o resistencia al deslizamiento/derrape se mide mediante el péndulo de fricción TRRL o péndulo británico (UNE-EN 13036-4:2012) (Figura 1). La medida de este péndulo puede emplearse en vías ciclistas, pero tiene el inconveniente de que es un ensayo lento, con escasa reproducibilidad y con el que se obtienen medidas puntuales.

El Ministerio de Fomento de España valora la resistencia al deslizamiento en carreteras de mezclas bituminosas mediante el coeficiente de rozamiento transversal (CRT) (NLT-336/92) medido mediante el SCRIM (Sideway-force Coefficient Routine Investigation Machine). Sin embargo, al ser un equipo muy caro se han desarrollado equipos alternativos, entre los que destaca el GRIPTESTER o el PAVETESTING CFT. El primero mide el coeficiente de rozamiento longitudinal denominado Grip Number (GN), correlacionado con el CRT. El principio de funcionamiento del segundo equipo es similar al SCRIM, y su uso está normalizado en EE. UU. [ASTM E670], pero ninguno de estos equipos, que son desarrollados para carreteras, puede utilizarse en vías ciclistas debido principalmente a sus dimensiones (9) (10), a excepción de una variante del GRIP-

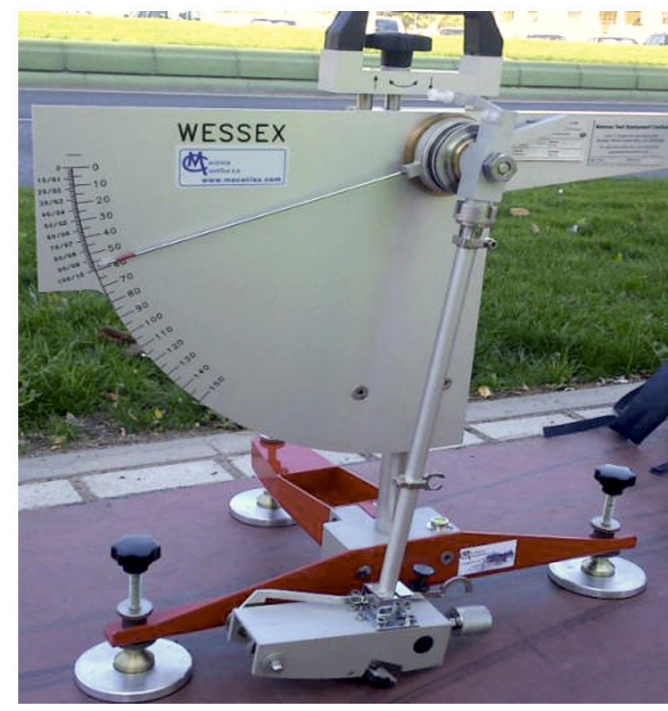

a) Péndulo TRRL

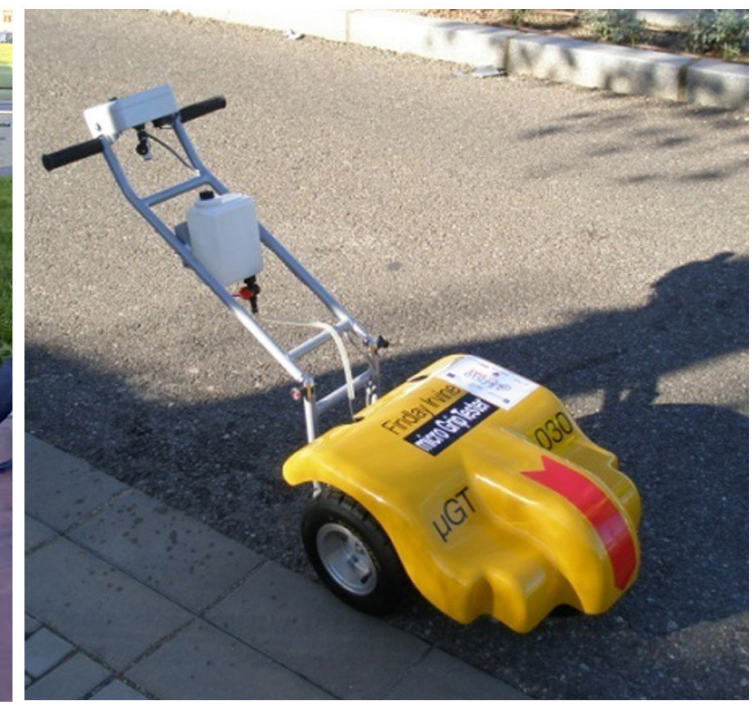

b) MICROGRIPTESTER

Figura 1. Equipos de medida de la resistencia al deslizamiento/derrape en vías ciclistas 
TESTER denominado MICROGRIPTESTER desarrollado por Findlay Irvine LMT (http://www.findlayirvine.com) (Figura 1). El equipo mide con una rueda normalizada según la ASTM E1844, orientada en la dirección de avance y parcialmente bloqueada (15\%). Es versátil y ligero. Toma datos cada $25 \mathrm{~mm}$ a una velocidad de $0,7 \mathrm{~m} / \mathrm{s}$ a $1 \mathrm{~m} / \mathrm{s}$. Permite un ajuste de la lámina de agua de $0,25 \mathrm{~mm} 1 \mathrm{~mm}$.

Por otro lado, la regularidad superficial es un parámetro que se utiliza en firmes de carreteras para determinar la comodidad en la conducción. En la práctica se ha comprobado, que si las vías ciclistas son incómodas, algunos ciclistas prefieren circular por la calzada, compartiendo espacios con los vehículos motorizados, aun cuando esto puede suponer sacrificar su seguridad a cambio de comodidad (11).

La medida de la regularidad superficial puede hacerse por dos grupos de métodos. El primero está formado por los que utilizan referencias geométricas, entre el que destaca la «regla de $3 \mathrm{~m}$ » (en Alemania, de $4 \mathrm{~m}$ ) por la cual se mide la distancia máxima en mm entre la capa y la regla, colocando ésta en cualquier punto y dirección (NLT-334/87). El segundo grupo es el formado por los sistemas de medida de tipo dinámico, como el Analizador de la Regularidad Superficial (ARS) (3).

En carreteras, a fin de poder comparar las medidas realizadas con equipos diferentes, se ha impuesto el empleo del Índice de Regularidad Internacional (IRI) medido según la ASTM E1926-08, que emplea un modelo matemático (Qcs) que simula la suspensión y masas de un vehículo tipo circulando por un tramo de carretera a una velocidad de $80 \mathrm{~km} / \mathrm{h}(3)$. Por definición, este índice no tiene aplicación en vías ciclistas, y además, se necesitan vehículos instrumentalizados cuyo uso en éstas es impracticable, por lo que como alternativa, han surgido otros equipos más pequeños como el Walking Profiler. El Walking Profiler es un equipo compacto desarrollado por ARRB Group Ltd (https://www.arrb.com.au) para la medida continua del perfil, pendiente, distancia e IRI. Cumple con los requisitos de perfilómetro de tipo World Bank Class 1. Las medidas se realizan entre 1 y $1,5 \mathrm{~m} / \mathrm{s}$.

La consonancia entre adherencia y regularidad superficial debe marcar el nivel óptimo de rugosidad superficial (12), evitando superficies que generen exceso de vibraciones y reduzcan la comodidad. Otros factores como la ubicación de rejillas y tapas de registro, la estética, la presencia de raíces, la climatología, etc., son elementos a tener en cuenta en el diseño del pavimento (13), así como proveer áreas peatonales adecuadas y suficientes para evitar la presencia de peatones en las vías ciclistas (14).

En España existe una carencia de criterios para el diseño de pavimentos en vías ciclistas, por lo que cada Comunidad Autónoma o ciudad ha establecido unos criterios propios. El presente artículo presenta los resultados de un estudio bibliográfico llevado a cabo en el marco del Proyecto ciclovíAs relativo a las especificaciones de adherencia y regularidad superficial de vías ciclistas.

\section{TIPOS DE PAVIMENTOS}

Los materiales más sugeridos y utilizados para la construcción de pavimentos en vías ciclistas son las mezclas bituminosas y el hormigón (5) (15), que representan aproximadamente el 80 y $10 \%$ respectivamente (16).

\subsection{Mezcla bituminosa}

El artículo 542 del PG-3 (17) define la mezcla bituminosa en caliente tipo hormigón bituminoso (MBC-AC) como la combinación de un ligante hidrocarbonado, áridos (incluido el polvo mineral) con granulometría continua y, eventualmente, aditivos, de manera que todas las partículas del árido queden recubiertas por una película homogénea de ligante. Su puesta en obra se realiza a temperatura muy superior a la del ambiente, entre unos 140 y $160{ }^{\circ} \mathrm{C}$. Son similares a las empleadas en la construcción de carreteras, cuyas características se recogen en el PG-3 (17) y cuya tecnología de ejecución es bastante conocida (5).

Entre las ventajas de su uso, destaca que los pavimentos bituminosos proporcionan una gran regularidad superficial ofreciendo al ciclista una gran comodidad (9) (18) (19) (20), buena adherencia, y durabilidad (13).

Su empleo es adecuado en áreas susceptibles a movimientos del terreno, y proporciona una superficie más suave en los casos de deformaciones por raíces de los árboles (9) (20). Es la superficie preferida, sobre todo para rutas de alto tráfico ciclista (21), y generalmente van acompañadas de un tratamiento superficial, en cuyo caso tendrán menos costes de mantenimiento a largo plazo (21).

Además, tienen la posibilidad de colorarse mediante el empleo de colorantes en el aglomerado de la capa de rodadura (19) aunque su coste es mucho mayor (19) (22) por lo que en la mayoría de los casos la coloración se obtiene con un tratamiento superficial. Se propone el color rojo, mediante óxido de hierro, (4) o verde, mediante óxido de cromo (18) en pavimentos asfálticos de zonas urbanas, y negro en el resto de los casos por motivos económicos (4). En todo caso, la coloración debe ir complementada con la demarcación y señalización horizontal para el acabado final (5).

Las principales desventajas de las mezclas bituminosas en caliente son:

- Vida más corta (7-20 años) que en pavimentos de hormigón (20) (23).

- Mayores exigencias de mantenimiento en comparación con el hormigón (2) (20) (23).

- Puesta en obra más laboriosa (20) que en obra de hormigón.

- No hay diferencia de color entre la vía ciclista y la calzada motorizada. La coloración encarece el producto más que en hormigón (20).

- Crecimiento de hierba y malas hierbas a través del pavimento. Requiere tratamiento regular con herbicida para prevenir el crecimiento de hierba a partir de los nutrientes del betún (22).

- Riesgo de roderas en el pavimento por las altas temperaturas del verano, si no se utiliza un ligante adecuado o una dotación adecuada del mismo (20).

Se recomienda que la mezcla de asfalto tenga menos del $7 \%$ de huecos (mezcla graduada densa). Sin embargo, si es posible, el contenido de betún debe aumentarse ya que las cargas estimadas serán más ligeras que en carreteras (20). El espesor recomendado es de $5 \mathrm{~cm}$, extendida en una sola capa sobre una superficie muy regular y bien compactada. En caso de una capa más irregular puede admitirse el extendido en dos 
capas de 4 y $3 \mathrm{~cm}$ (total $7 \mathrm{~cm}$ ) (18). La extensión de las capas de mezcla bituminosa sobre zahorra debe ir precedida de un riego de imprimación, y entre capas de mezcla bituminosa es necesario un riego de adherencia.

El tamaño máximo de árido utilizado suele ser de $16 \mathrm{~mm}$, aunque en los casos en que la mezcla se extienda en dos capas, de 4 y $3 \mathrm{~cm}$, o incluso menos, habría que recurrir a mezclas con tamaño máximo de árido de $8 \mathrm{~mm}$. En cuanto al ligante, la elección del mismo debe hacerse teniendo en cuenta la zona climática donde se ubique la vía ciclista. Así, en zonas con temperaturas elevadas, suele utilizarse betún de penetración B35/50, para evitar el riesgo de deformaciones plásticas; y en zonas con bajas temperaturas, es preferible la elección de un betún 50/70 para prevenir problemas de fisuraciones térmicas.

Además de las mezclas bituminosas en caliente, se deben considerar también las mezclas bituminosas abiertas en frio (MAF) fabricadas a partir de emulsión bituminosa y cuya fabricación, almacenamiento, extendido y compactación se realiza a temperatura ambiente (4), así como las técnicas de mezclas semicalientes o templadas (MBT-AC) cuyo uso reduce significativamente las emisiones de $\mathrm{CO}_{2}$, minimiza los consumos energéticos y conlleva una mejora de las condiciones de trabajo, seguridad y salud laboral (24). En los Proyectos de nuevas vías ciclistas de la Consejería de Fomento y Vivienda de la Junta de Andalucía del año 2014 se han empleado mezclas tipo MBC AC 16 SURF (s) y MBT AC 16 SURF(s).

\subsection{Hormigón}

Se trata de losas de hormigón en masa separadas por juntas transversales, o por una losa continua de hormigón armado, en ambos casos eventualmente dotados de juntas longitudinales (17). Las juntas de retracción están diseñadas para controlar la fisuración, mientras que las juntas de dilatación, para permitir el movimiento térmico del pavimento. Deben estar colocadas perpendicularmente a la dirección de la vía para minimizar la incomodidad de los ciclistas (20).

El sellado de juntas mejora la comodidad del usuario, proporcionando una transición más suave entre las losas y evitando que la suciedad y el agua entre en las juntas.

Los requisitos de los pavimentos de hormigón deben ser: pocas juntas y simples, resistencia al deslizamiento en condiciones secas y mojadas y con un bajo mantenimiento (20). Se recomienda un acabado de superficie para proporcionar una resistencia al deslizamiento adicional para ciclistas, particularmente en condiciones de humedad (20) (21). Esto puede lograrse mediante un cepillado transversal o estriado sobre el hormigón fresco, o granallado o fresado ligero sobre el hormigón endurecido.

También se pueden utilizar pigmentos inorgánicos para mejorar la apariencia y minimizar el deslumbramiento (20). Es posible la terminación mediante tratamiento fratasado con coloreador superficial, cuarzo y/o endurecedor (18), aunque este método aumenta el riesgo al deslizamiento/derrape.

Entre las ventajas que presenta este material destacan:

- Pavimento durable con excelentes condiciones (20) (21) (25).
- Menor resistencia a la rodadura en comparación con las mezclas bituminosas y superficies no pavimentadas (2) (20) (22) (23) (25).

- Mayor vida útil (algunas veces sobre 40 años) (9) (23), necesitando un mantenimiento muy inferior al del pavimento bituminoso (2) (22) (25).

- Facilidad de ejecución (2) (20) (22).

- Posibilidad de cambiar el color y la textura mediante diferentes acabados de superficie (4) (18) (20).

- Más fácil de extender y enrasar alrededor de pozos o arquetas de registro (20).

- Menor susceptibilidad a la deformación de las raíces y las malas hierbas (4) (18), aunque si se produce, puede causar separación vertical entre losas de hormigón (20).

Sin embargo, posee una serie de inconvenientes como los que se detallan a continuación:

- Discontinuidad debida a las juntas de construcción y dilatación (9) (20) (23).

- Mayor coste de construcción y reparación (13) (19) (20) (21) en comparación con las mezclas bituminosas (6).

- Las marcas viales en el pavimento no son tan visibles como lo son sobre el asfalto, especialmente durante la noche (23).

- Pueden producirse fisuras (o incluso roturas) de la losa por las variaciones climáticas o movimientos del terreno natural o retracción (22).

- Las juntas requieren un control para evitar vegetación entre losas (20).

- Para maximizar la suavidad, el relleno de las juntas de dilatación debe mantenerse justo por debajo de la superficie acabada (20).

El hormigón impreso es un tipo de pavimento de hormigón al que se le aplica un tratamiento superficial mediante el sistema de estampar, texturizar y colorear in situ el hormigón fresco. En los últimos años se han desarrollado muchas opciones con las que se disponen de texturas y colores muy diversos pero lleva implícito una pérdida de regularidad superficial (19).

El tipo de pavimento de hormigón más utilizado en los proyectos de vías ciclistas de la Conserjería de Fomento y Vivienda de Andalucía del año 2014 fue el hormigón vibrado HF3,5 , en continuo, en un espesor de $14 \mathrm{~cm}$ (18), aplicado con un revestimiento superficial rugoso con resina acrílica para dar coloración a la vía y mejorar la adherencia.

\subsection{Tratamientos superficiales}

Dotan al firme de determinadas características superficiales, pueden distinguirse dos tipos de tratamientos superficiales:

a) Riegos con gravilla: se componen de una mezcla de ligante hidrocarbonado (betún fluidificado o emulsión) y gravilla uniforme (18) (19) (22), empleándose para restituir las propiedades superficiales del firme e incluso como capa de rodadura en firmes rurales o de escaso tráfico rodado. Tienen un coste de ejecución normalmente bajo, pero suelen requerir mayor mantenimiento por desprendimiento de áridos (4). Se clasifican en riegos monocapa, riegos bicapa, riegos monocapa de doble engravillado, riegos sándwich, o riegos multicapa.

En los proyectos de la Consejería de Fomento y Vivienda de la Junta de Andalucía no se han contemplado los 
tratamientos superficiales de riego con gravilla en las vías ciclistas urbanas, aunque sí están contemplados de forma genérica en los esquemas de firmes propuestos en las Recomendaciones de diseño de vías ciclistas de la mencionada administración (18).

b) Microaglomerados en frío (lechadas bituminosas): formadas por una mezcla a temperatura ambiente de una emulsión bituminosa, áridos finos, agua y, eventualmente, polvo mineral y otros productos como fibras o derivados de la trituración de neumáticos. Se utilizan como tratamiento superficial de mejora de la textura y resistencia al deslizamiento (18). Su empleo en nuestro país está muy extendido, denominándose de manera genérica slurrys (4) (19). Los espesores habituales no suelen ser mayores a $3 \mathrm{~mm}$.

c) Tratamientos con resinas acrílicas: Se trata de un número variable de capas (entre 1 y 5 capas) aplicadas sobre el firme de mezcla bituminosa o de hormigón, aunque lo más utilizado es un sistema de 3 capas. Confieren adherencia y un aspecto estético diferenciador. En el caso de mezcla bituminosa, los fabricantes recomiendan dos capas de mortero sintético con una dotación de $1 \mathrm{~kg} / \mathrm{m}^{2}$ y una capa de pintura final de sellado. En el caso de hormigón, una capa que actúe de adherencia con una dotación de $0,5 \mathrm{~kg} / \mathrm{m}^{2} \mathrm{y}$ una capa de mortero sintético de $1 \mathrm{~kg} / \mathrm{m}^{2}$ más una última capa de pintura de sellado. La experiencia ha puesto de manifiesto que el número de capas de resina epoxi acrílica puede reducirse a dos, con lo cual se ahorran costes sin disminuir las prestaciones de la capa de rodadura. Las nuevas vías ciclistas de la Consejería de Fomento y Vivienda de la Junta de Andalucía proyectadas en 2014 utilizan dos capas de resinas epoxi acrílicas sobre la mezcla bituminosa.

\subsection{Adoquines o baldosas}

El pavimento de adoquines o baldosas tiene un coste de ejecución y mantenimiento superior a la mezcla bituminosa (13) (19). La conducción no es tan cómoda por la discontinuidad de los elementos, por lo que suele emplearse en tramos cortos y por motivos estéticos o de integración paisajística en vías urbanas, o en zonas donde es necesario reducir la velocidad de los ciclistas (5) (14) (22). Para que las discontinuidades no adquieran un carácter longitudinal, las baldosas han de tener una disposición transversal en aras de una mayor seguridad (2).

Se prefiere los prefabricados de hormigón de unos $6 \mathrm{~cm}$ de espesor para el caso del adoquín y $4 \mathrm{~cm}$ para el caso de la baldosa (4) (12) (18). Se colocarán sobre un lecho de arena $\mathrm{o} / 6 \mathrm{~mm}$ de $3 \mathrm{~cm}$ de espesor, de naturaleza silícea y el relleno de juntas se hará con arena fina o mortero (12).

\subsection{Materiales sueltos: gravas, suelos y zahorras}

Las superficies no pavimentadas sólo son apropiadas en vías ciclistas con uso recreativo (5), cada vez más demandadas por los usuarios de bicicletas de montaña (15) o bien como medida provisional antes de obtener la financiación correspondiente para la pavimentación (23). En estos casos, los materiales deben ser firmes y estables como grava, zahorra artificial, suelo estabilizado y piedras calizas, dependiendo de la disponibilidad local (23).
En el caso de las gravas, tiene menor precio y menor coste de construcción inicial, pero con coste más elevado de mantenimiento (nivelación, barrido, y control de malezas) y muy incómodo de conducción, sobre todo en tiempo lluvioso (20). Los suelos tienen un coste inicial bajo pero se requiere un alto mantenimiento para conservar una superficie de rodadura adecuada, y el drenaje es a menudo un problema (20). El uso de zahorras está muy limitado a entornos naturales (4) (18) (25) debido a su gran integración visual. Se trata de materiales económicos pero con poca adherencia debido a la falta de cohesión de las partículas (19), poca durabilidad, erosionables, y que favorece la aparición de vegetación (18).

La zahorra caliza de granulometría uniforme es fácil de ejecutar y mantener, no se agrieta y en general proporciona una superficie cómoda de conducción, integrándose bien visualmente en entornos naturales. Sin embargo, pierde la cohesión de sus partículas con el tiempo, aumentando así el riesgo de derrape de la bicicleta. También está sujeto a la erosión y la invasión de vegetación. En tiempo seco, el aumento de polvo puede dañar los mecanismos de la bicicleta y provocar una conducción incómoda. Pendientes de más del $3 \%$ no deben estar recubiertos de piedra caliza triturada, porque la superficie no proporcionará suficiente tracción (25).

En estos últimos años, es común la propuesta de usar áridos reciclados de construcción y demolición en la construcción de vías de baja intensidad de tráfico (26) (27). Estos materiales tienen las mismas ventajas que la zahorra caliza de granulometría uniforme. Se proponen para su uso en la construcción de base de firme, aunque no para pavimentos/capas de rodadura (18), que es nuestro objeto de estudio.

\subsection{Suelo-cemento}

El suelo cemento también tiene aspecto natural y un coste económico bajo, tiene una buena adherencia, por lo que se puede usar en entornos naturales y otros no muy exigentes con la calidad final del acabado (4) (18). Esto es así ya que se trata de un material erosionable, con poca durabilidad, con alta necesidad de mantenimiento y con mala calidad superficial (18).

\subsection{Otros pavimentos especiales y marcas viales}

En zonas protegidas se utilizan otros «pavimentos especiales» como bases granulares compactadas, suelos estabilizados, resinas especiales, etc. (18).

En algunos casos concretos, como en pasos sobre puentes, se utilizan pavimentos de madera, aunque su uso no es usual.

Otro elemento a considerar en el pavimento de vías ciclistas por su incidencia en el riesgo de deslizamiento, y por tanto en la seguridad, es la marca vial, reflectorizada o no, que se define como «aquella guía óptica situada sobre la superficie de la calzada o vía ciclista, formando líneas o signos, con fines informativos y reguladores del tráfico». En la aplicación de éstas marcas se utilizarán pinturas, termoplásticos de aplicación en caliente, plásticos de aplicación en frío, o marcas viales prefabricadas (17). Pueden ser convencionales (tipo 1), o con resaltes u otros diseños específicos (tipo 2) para mantener sus propiedades en condiciones de lluvia o humedad. 


\section{ELECCIÓN DE TIPO DE PAVIMENTO}

Como se ha dicho anteriormente, en la elección del tipo de pavimento hay que considerar las características superficiales, buscando un equilibrio entre seguridad (adherencia) y comodidad (regularidad superficial), así como los criterios económicos en la ejecución y conservación y mantenimiento (18).

Teniendo en cuenta toda esta información, las mezclas bituminosas, hormigón, baldosas o adoquines son empleados mayoritariamente en zonas urbanas (2), aunque en cascos históricos o algunas zonas urbanas singulares se suele emplear adoquín, baldosas, losas de piedra natural u hormigón impreso, reforzando criterios como los de tipo estético o visual (4), si bien no son los más adecuados desde el punto de vista económico y funcional.

En las zonas donde la vía ciclista es parte de la calzada de las carreteras (zonas interurbanas), se debe asegurar una conducción cómoda para evitar que los ciclistas invadan los carriles de tráfico (17). Para ello, se recomienda el uso de mezclas bituminosas, delimitados o coloreados para distinguirse de las superficies adyacentes o cunetas.

Para evitar el impacto visual del asfalto y el hormigón en espacios naturales o caminos rurales se puede optar por un pavimento formado por material granular compactado o estabilizado, aunque hay que considerar que aumenta la resistencia a la rodadura, incrementando el esfuerzo y reduciendo la velocidad del ciclista (2).

\section{REGULARIDAD SUPERFICIAL}

El criterio de comodidad exige una superficie uniforme con ausencia de baches, protuberancias o discontinuidades que puedan afectar a la estabilidad de la bicicleta (2) (13). Así, hay varios factores de diseño y construcción que afectan a la comodidad y transitividad de las vías ciclistas (20):

- Las superficies rugosas u onduladas, causadas por el uso de áridos grandes, y/o la existencia de baches, raíces expuestas, rejillas, desagües, etc

- La presencia de gravilla suelta, arena o barro que dificulta el tránsito y disminuye además la adherencia

- Drenaje superficial deficiente

En el caso de que existan discontinuidades, estas deberán ser de reducidas dimensiones, de tal forma que no afecten a la rodadura del neumático y garanticen una conducción favorable (2) (4) (28).

Debe evitarse la colocación de alcantarillas, tapas de pozos de registro y arquetas, así como otras irregularidades sobre la vía ciclista, pero si esto fuera inevitable, se debe asegurar que estén nivelados con la superficie de la vía (2) (5) (20). Las juntas, en caso de pavimentos rígidos, deben ser selladas (5) y en buenas condiciones, y se debe garantizar la retirada de arena, tierra, suciedad y otros elementos que puedan causar accidentes.

En el caso de pavimentos de hormigón, debe asegurarse que a los 5 años de vida útil, los desplazamientos verticales sean menores de $15 \mathrm{~mm}$ (20). Un estudio realizado por una consultora del transporte por carretera de Australia (ARRB Trans- port Research) (20) mostró que muy pocos ciclistas sintieron «incomodidad» con desplazamientos verticales entre losetas-baldosas de $6 \mathrm{~mm}$, más de la mitad sintieron «algo de incomodidad» con $12 \mathrm{~mm}$ y todos sintieron «incomodidad» con $18 \mathrm{~mm}$ (20).

La orientación de las rejas de drenaje debe ser perpendicular al sentido de la circulación (2) y las diferencias de altura debida a tapas de alcantarillas y otros resaltos o bordillos no deben exceder los $5 \mathrm{~mm}$ (20). En los casos en los que se requieran ranuras y peldaños, para reducir los peligros potenciales de que la rueda de la bicicleta se introduzca en éstas, se propone una anchura máxima de la ranura de $12 \mathrm{~mm}$ (9) (20) $-12,7 \mathrm{~mm}$ en (29) - y una altura de $10 \mathrm{~mm}$ (9) (20), paralelamente a la conducción, y $20 \mathrm{~mm}$ de altura del peldaño para las ranuras perpendiculares a la dirección de la conducción (9) (20). En asfalto, se limita a un $10 \%$ las fisuras de más de $8 \mathrm{~mm}$ de ancho (20). Los espacios entre la rejilla y su marco no deben exceder los 6,4 $\mathrm{mm}$ (30).

Para asegurar una superficie segura y cómoda y evitar los costes de mantenimiento y reconstrucción, se recomiendan unas actividades rutinarias de conservación como el sellado de grietas, nivelado de baches, limpieza de las vías ciclistas y repintado de la señalización horizontal (20) (31). Mientras se realice estas operaciones de conservación, se debe avisar al peatón-ciclista mediante señalización y es necesario proporcionar una ruta alternativa temporal (20).

Sin embargo, el usuario considera que las superficies rugosas y caminos estrechos son los problemas más importantes que se encuentran con mayor frecuencia (20). Por otro lado, los baches y superficies resbaladizas son problemas muy importantes, pero son menos frecuentes. Normalmente las fisuras en la superficie son consideradas un problema menor (20).

La regularidad superficial de cada tipo de pavimento queda resumida en la Tabla 1, indicando la recomendación nacional o internacional del que se ha obtenido. En el caso del hormigón, es recomendable disponer de juntas de retracción transversal cada 4 o $5 \mathrm{~m} \mathrm{(18)} \mathrm{(22),} \mathrm{ejecutadas} \mathrm{por} \mathrm{corte} \mathrm{del} \mathrm{pavimento} \mathrm{en-}$ tre las 6 y 24 horas de la puesta en obra del hormigón, con una profundidad comprendida entre $1 / 4$ y $1 / 3$ del espesor de la losa (2) (12), estas juntas provocan cierta incomodidad de rodadura para el usuario. Las juntas de dilatación se diseñan para permitir el movimiento térmico del pavimento, han de seccionar el espesor completo de la solera y se recomiendan juntas de dilatación cada $20 \mathrm{~m}$ con anchuras inferiores a $1 \mathrm{~cm}$ y rellenas de un material compresible (2). Otras guías (12) sólo indican que la anchura y el número de juntas sea la mínima posible.

Por esta razón, la mayoría de guías y pliegos exponen una regularidad superficial «buena» para este material, aunque condicionada a estas juntas.

Igualmente, aristas como las que se pueden encontrar en la unión entre el pavimento y una alcantarilla, no deben exceder los 9,5 mm de alto en dirección de la conducción ni 19,1 mm en dirección perpendicular para evitar que los ciclistas pierdan el control en dichas zonas (29).

El Caltrans Gighway Design Manual, sin embargo, especifica tolerancias más estrictas en estas superficies (29). Así, el punto más bajo de un tramo de aproximadamente $2,5 \mathrm{~m}$ de largo no debe diferir más de $6 \mathrm{~mm}$ en cualquier dirección. 
La Tabla 2 muestra los criterios para calificar una vía ciclista de mezcla bituminosa u hormigón según la guía Australiana (9). Sobre las marcas viables, deben ser visibles, y no resbaladizas, ni elevarse más de $4 \mathrm{~mm}$ sobre el pavimento (23).

En toda la bibliografía española consultada, no se ha encontrado métodos para medir la regularidad superficial, excepto en Andalucía (18), que propone la regla de los $3 \mathrm{~m}$ aunque no se especifi- can los valores aconsejables de ésta, lo cual justifica la necesidad de establecer un criterio de medida de esta propiedad.

En cuanto a la documentación internacional, la ARRB Transport Research mide la rugosidad a través del IRI usando el Walking Profiler, y limita su valor a $3,5 \mathrm{~m} / \mathrm{km}$ a los 5 años (equivalente a $20 \%$ de insatisfacción) y a $8 \mathrm{~m} / \mathrm{km}$ a $\mathrm{los} 20$ años (equivalente a $50 \%$ de insatisfechos) (20).

Tabla 1. Regularidad superficial de vías ciclistas según tipo de pavimento.

\begin{tabular}{|c|c|c|c|c|c|c|c|c|}
\hline & \begin{tabular}{|c|} 
Mezcla \\
bituminosa
\end{tabular} & $\begin{array}{l}\text { Tratamientos } \\
\text { superficiales }\end{array}$ & Hormigón & Adoquines & Baldosas & \begin{tabular}{c|} 
Materiales \\
sueltos
\end{tabular} & $\begin{array}{l}\text { Suelo-ce- } \\
\text { mento }\end{array}$ \\
\hline \multirow{7}{*}{ 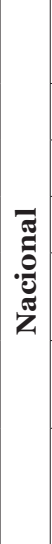 } & Andalucía (18) & Buena & Aceptable & $\begin{array}{l}\text { Buena (condicio- } \\
\text { nada a las juntas) }\end{array}$ & \multicolumn{2}{|c|}{$\operatorname{Regular}^{*} 1$} & \multicolumn{2}{|c|}{ Regular } \\
\hline & Cataluña (20) & Buena & - & - & \multicolumn{2}{|c|}{-} & \multicolumn{2}{|c|}{-} \\
\hline & Guipúzcoa (19) & Buena & - & Según textura & Baja & Baja & \multicolumn{2}{|c|}{ Rodadura suave } \\
\hline & Málaga (12) & Buena & Aceptable & Buena & Regular & Regular & \multicolumn{2}{|c|}{-} \\
\hline & \begin{tabular}{|l|} 
Ministerio \\
Fomento (13) \\
\end{tabular} & Buena & - & Buena & Baja & Baja & \multicolumn{2}{|c|}{-} \\
\hline & Vitoria-Gasteiz (4) & Buena & - & Según textura & Baja & Baja & $\begin{array}{l}\text { Rodadura } \\
\text { suave }\end{array}$ & Buena \\
\hline & Zaragoza (2) & Buena & - & Buena & Baja & $\begin{array}{c}\text { Buena } \\
\text { (según tipo } \\
\text { de baldosa) }\end{array}$ & $\begin{array}{l}\text { Regular } \\
\text { (según } \\
\text { entorno) }\end{array}$ & - \\
\hline \multirow{2}{*}{ 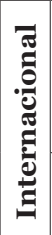 } & Francia (32) & Muy buena & Aceptable & $\begin{array}{l}\text { H. liso: Buena } \\
\text { H. estriado: } \\
\text { Aceptable }\end{array}$ & $\begin{array}{l}\text { Regular- } \\
\text { Baja }\end{array}$ & Regular & - & Regular \\
\hline & Londres (28) (33) & Buena & Buena & Buena & Aceptable & Aceptable & - & - \\
\hline
\end{tabular}

Tabla 2. Calificación de las vías de mezclas bituminosas y hormigón (9).

\begin{tabular}{|c|c|c|}
\hline Calificación & Mezclas bituminosas & Hormigón \\
\hline \multirow{4}{*}{$\begin{array}{c}\text { Poco } \\
\text { favorable }\end{array}$} & Grietas longitudinales y transversales de la superficie $>10 \mathrm{~mm}$ de ancho & \multirow{2}{*}{$\begin{array}{l}\text { Grietas en el pavimento; el hormigón está } \\
\text { suelto, disgregado o con áridos sueltos }\end{array}$} \\
\hline & Agrietamiento del pavimento tipo «piel de cocodrilo» & \\
\hline & $\begin{array}{l}\text { Grietas longitudinales de pavimento con malas hierbas generalmente } \\
\text { situadas en el borde la vía en combinación con fallo del pavimento }\end{array}$ & $\begin{array}{l}\text { Grietas anchas }(>10 \mathrm{~mm}) \text { con desplazamiento } \\
\text { vertical }>25 \mathrm{~mm}\end{array}$ \\
\hline & $\begin{array}{l}\text { Ondulaciones de la superficie por desgaste o erupciones }<20 \mathrm{~mm} \text {. } \\
\text { Generalmente debido a daños de raíces deárboles o reacciones químicas } \\
\text { (hinchamientos) de la capa base }\end{array}$ & Desniveles severos de la acera \\
\hline \multirow{5}{*}{ Tolerable } & Grietas longitudinales o transversales de la superficie $<10 \mathrm{~mm}$ de ancho & \multirow[b]{2}{*}{$\begin{array}{l}\text { Grietas }>10 \text { mm con desplazamiento hori- } \\
\text { zontal del hormigón }<25 \mathrm{~mm}\end{array}$} \\
\hline & $\begin{array}{l}\text { Tramos aislados perceptibles de áreas continuas de agrietamiento tipo } \\
\text { «piel de cocodrilo» }\end{array}$ & \\
\hline & $\begin{array}{l}\text { Tramos perceptibles de longitudes continuas de pavimento con malas } \\
\text { hierbas, generalmente situadas a lo largo del borde la vía, en combina- } \\
\text { ción con fallo del pavimento }\end{array}$ & Resaltos $<30 \mathrm{~mm}$ \\
\hline & Ondulaciones de la superficie por desgaste o erupciones $<20 \mathrm{~mm}$ & \\
\hline & \multicolumn{2}{|c|}{$\begin{array}{l}\text { Comentario genérico: un pavimento tolerable pasa a ser poco favorable cuando se requiera una intervención para preservar } \\
\text { la comodidad y/o garantizar la seguridad }\end{array}$} \\
\hline \multirow{3}{*}{ Buena } & Vías con calidad de rodadura satisfactoria & $\begin{array}{l}\text { Grietas pequeñas-medianas }<10 \mathrm{~mm} \text { de ancho, } \\
\text { y grietas longitudinales sin o con pequeño des- } \\
\text { plazamiento vertical }\end{array}$ \\
\hline & Deterioros pequeños de superficie que no requieren intervención & Resaltos $<30 \mathrm{~mm}$ \\
\hline & \multicolumn{2}{|c|}{$\begin{array}{l}\text { Comentario genérico: una buena condición se da cuando el pavimento no está deteriorado ya que no requiere trabajos } \\
\text { de mantenimiento }\end{array}$} \\
\hline Excelente & \multicolumn{2}{|c|}{ Vías nuevas, sin grietas, ni ondulaciones, ni deterioros observables, y que no requiere trabajos de mantenimiento } \\
\hline
\end{tabular}


En este mismo estudio (20), se obtuvieron correlaciones razonablemente buenas entre aceptabilidad e IRI para pavimentos asfálticos, siendo un IRI de $4 \mathrm{~m} / \mathrm{km}$ equivalente a una calificación «buena» para el $75 \%$ de usuarios. De igual forma, todas las vías de hormigón, sin importar la rugosidad medida por el IRI, tuvieron muy buenas calificaciones de aceptabilidad. Sin embargo, de forma general, se obtuvieron correlaciones muy bajas entre IRI y aceptabilidad, por lo que no se recomienda el uso de esta medida en vías ciclistas.

\section{ADHERENCIA}

Centrándonos en el pavimento, y para una correcta seguridad, se exige que éste tenga una textura superficial adecuada, ofreciendo resistencia al deslizamiento en cualquier circunstancia, fundamentalmente con pavimento mojado (22) y en trayectorias curvas (4).

En zonas con precipitaciones intensas es necesario tener en consideración el drenaje superficial de la vía ciclista. Se debe proporcionar una rápida evacuación del agua de lluvia para permitir seguridad y comodidad en la conducción, evitar la pérdida de adherencia de la bicicleta, así como el deterioro del pavimento (18). Esto se consigue mediante el uso de una pendiente transversal adecuada para eliminar el encharcamiento de agua en la superficie y bocas de alcantarillado fuera de la vía, al final de esta pendiente (20). Así, se recomienda una pendiente del $2 \%$ en la superficie y evitar pendientes en los dos sentidos (23). De forma similar, en los peraltes, se aconseja una inclinación mínima del $2 \%$, necesario para estimular el drenaje adecuado, y un máximo aproximado de $5 \%$, por encima del cual se pueden encontrar dificultades en la conducción (15).

El coeficiente de fricción depende de la velocidad de la bicicleta, tipo de superficie, rugosidad, tipo y estado de los neumáticos, temperatura y si la superficie está húmeda o seca. Extrapolando los valores utilizados en el diseño de carreteras, los factores de fricción para pavimentos de vías ciclistas pueden variar de 0,30 a $23 \mathrm{~km} / \mathrm{h}$ a 0,22 a $50 \mathrm{~km} / \mathrm{h}$ (15). Aunque no hay datos disponibles para las superficies no pavimentadas, se sugiere que los factores de fricción se reduzcan en un $50 \%$ para permitir un margen de seguridad suficiente (15).

La adherencia según los tipos de pavimentos se muestra en la Tabla 3. De estas se extrae que las mezclas bituminosas poseen «buena» o «muy buena» adherencia, ofrecen poca resistencia a la rodadura y una resistencia «razonable» al deslizamiento (2). No se erosionan y son duraderos, siempre y cuando se hayan dimensionado y ejecutado correctamente.

En las recomendaciones de Andalucía (18), Zaragoza (2) y Málaga (12) se expone una «buena» adherencia para los pavimentos de hormigón, mientras que el Ministerio de Fomento (13) lo clasifica como "adecuada» (Tabla 3). Se advierte una especial atención al acabado de la superficie de rodadura, que debe ser, en todo caso, antideslizante, por lo que se evitarán tratamientos superficiales como el pulido mediante fratasado en hormigón (2). En caso de utilizarse resinas acrílicas o epoxi, es recomendable determinar la adherencia del revestimiento al sustrato o capa sobre la que se aplica; lo que podrá efectuarse mediante la determinación de la resistencia a tracción sobre testigos extraídos del pavimento (18). Las recomendaciones consultadas no aportan ninguna especificación con respecto al valor aceptable de resistencia al deslizamiento de los pavimentos de hormigón (20).

Los materiales sueltos, poseen una rodadura variable según su estado de conservación, generalmente son suaves pero no muy adherentes, pues quedan partículas sueltas de material, si se erosiona con las lluvias se pueden producir roderas (4).

Los suelos tratados con cemento poseen mejor adherencia que los materiales sueltos, aunque no es muy duradera, ya que es sensible a los cambios de temperatura y de humedad (19).

Tabla 3. Adherencia en vías ciclistas según tipología de pavimento

\begin{tabular}{|c|c|c|c|c|c|c|c|c|c|}
\hline & & $\begin{array}{c}\text { Mezcla } \\
\text { bituminosa } \\
\end{array}$ & $\begin{array}{l}\text { Tratamientos } \\
\text { superficiales }\end{array}$ & Hormigón & \multicolumn{2}{|c|}{ Adoquines } & Baldosas & $\begin{array}{l}\text { Materiales } \\
\text { sueltos }\end{array}$ & $\begin{array}{c}\text { Suelo- } \\
\text { cemento }\end{array}$ \\
\hline \multirow{7}{*}{ 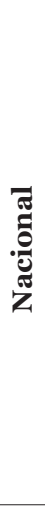 } & Andalucía (18) & Muy buena & Buena & Buena & \multicolumn{3}{|c|}{ Media $^{* 1}$} & \multicolumn{2}{|c|}{ Suficiente } \\
\hline & Cataluña (22) & Buena & - & Adecuada & \multicolumn{3}{|c|}{-} & - & - \\
\hline & Guipúzcoa (19) & Buena & Buena & Adecuada & \multicolumn{2}{|c|}{ Buena (prefabricados) } & $\begin{array}{c}\text { Mala } \\
\text { (cerámicos } \\
\text { o de piedra) }\end{array}$ & - & Baja \\
\hline & Málaga (12) & Buena & Buena & Buena & $*_{1}$ & Suficiente & & & \\
\hline & $\begin{array}{l}\text { Ministerio Fomento } \\
\text { (13) }\end{array}$ & Buena & - & Adecuada & \multicolumn{2}{|c|}{-} & - & - & - \\
\hline & Vitoria-Gasteiz (4) & Buena & - & - & $\begin{array}{c}\text { Buena } \\
\text { (pre- } \\
\text { fabricados) }\end{array}$ & $\begin{array}{c}\text { Mala } \\
\text { (cerámicos } \\
\text { o de piedra) }\end{array}$ & - & No alta & Buena \\
\hline & Zaragoza (2) & Muy buena & - & Buena & Media*1 & - & Muy buena & & \\
\hline 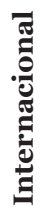 & & Buena & Buena & - & - & - & - & No Bueno & Buena \\
\hline
\end{tabular}

${ }^{* 1}$ Depende del material que haya sido usado para su fabricación y su grado de pulido. 
En cuanto a la medida de esta adherencia, a través de un estudio realizado en Nueva Zelanda, se concluye que los neumáticos de las bicicletas se comportan igual que los de los coches (20), y por lo tanto, los métodos aptos en neumáticos de bicicletas son:

- Ensayo del péndulo británico (BPN) (20). La resistencia al deslizamiento $R_{d}$, medida con este aparato debe ser mayor a 45 (18), medida con el patín 57 (corrección por temperatura distinta de $20{ }^{\circ} \mathrm{C}$ ). No más del $10 \%$ de las vías tendrán un deslizamiento menor de 60 a los 5 años (ya sea el pavimento de asfalto u hormigón), y asumiendo un tráfico mínimo, debería asegurarse un valor mínimo de deslizamiento de 50 para toda la vida útil de la vía (20).

- Grip Number (GN), determinado a partir del GRIPTESTER o MICROGRIPTESTER. La conversión aproximada de una medida a otra viene dada por la siguiente expresión:

$$
\mathrm{GN}=0,01 \times \mathrm{BPN}
$$

Así, en lugares difíciles (pendientes pronunciadas, curvas cerradas, paradas en semáforos, rotondas) se recomienda una velocidad máxima entre $60-80 \mathrm{~km} / \mathrm{h}$, correspondiente a un valor de GN entre 0,5 y 0,55 . Para carreteras principales urbanas y rurales, ambas con $\mathrm{GN}$ igual a 0,45 , se recomienda una velocidad máxima de 60 y $110 \mathrm{~km} / \mathrm{h}$, respectivamente, mientras que para ciclovías urbanas o con tráfico ligero, que es lo que pretende en este estudio, la velocidad máxima debe ser inferior a $60 \mathrm{~km} / \mathrm{h}$, con un GN igual a 0,40 (9).

En cuanto a la textura, para pavimentos de hormigón, y según el PG-3 (17) en su artículo 550.7, la profundidad de la textura superficial, determinada por el método del círculo de arena, según la NLT-335, deberá estar comprendida entre o,6 mm y 0,9 mm, aunque la ARRB Transport Research (20) indica un valor máximo de o,8 para asegurar buena comodidad en la conducción (el valor más alto encontrado en dicho estudio fue de o,6 en pavimentos de hormigón).

Tanto para mezclas bituminosas calientes (Art. 542.7), como para microaglomerados en frío (Art. 540.7), el PG-3 (17) exige un valor superior a $0,7 \mathrm{~mm}$ en este ensayo, mientras que la ARRB Transport Research (20) limita el valor máximo a 1,6 (en 5 años), por ser el valor máximo analizado que los usuarios calificaron como una conducción cómoda.

\section{CONCLUSIONES}

La resistencia al deslizamiento (adherencia) y la regularidad superficial son dos aspectos claves en la seguridad y comodidad de las vías ciclistas. Este trabajo de revisión ha puesto de manifiesto la carencia de normativas y recomendaciones de ámbito internacional, nacional, autonómico y/o local que establezcan una metodología de ensayo normalizada y unos valores límites de aceptación, rechazo y/o intervención en labores de conservación en vías ciclistas. Se han analizado un total de 50 documentos, entre normativas, guías, recomendaciones, libros, y artículos de investigación, sobre el diseño y construcción de vías ciclistas. Sólo 33 hacen referencia a materiales, resistencia al deslizamiento y regularidad superficial del pavimento.

A través de la bibliografía consultada, la medida de la regularidad superficial a través del IRI medido con el Walking Profiler puede ser una alternativa, limitando su valor a $3,5 \mathrm{~m} / \mathrm{km}$ a los 5 años y a $8 \mathrm{~m} / \mathrm{km}$ a los 20 años. Sin embargo, el índice IRI es un parámetro desarrollado para carreteras y de dudosa aplicación en vías ciclistas. La mayoría de las recomendaciones sólo hacen referencia a limitaciones en cuanto a irregularidades del pavimento como desplazamientos verticales de la rasante, anchuras y longitudes máximas de juntas, peldaños, interferencias (registros, rejillas).

En cuanto a la macrotextura del pavimento, se propone el ensayo del círculo de arena, con 0,7 de valor mínimo y 1,6 de máximo para pavimentos de mezclas bituminosas y de 0,6 y o,8, respectivamente, para pavimentos de hormigón.

La medición de la adherencia neumático-pavimento a través de la microtextura, puede realizarse mediante el ensayo del péndulo británico, limitando su valor, en todo caso, a un mínimo de 45 usando el patín 57, o a través del test Grip. El Micro-GripTester es un equipo desarrollado para la medida de marcas viales de carreteras que podría aplicarse en vías ciclistas con bastante éxito por su tamaño y versatilidad, sin embargo este ensayo no está normalizado, siendo necesarios ensayos que permitan armonizar el péndulo británico con el MICROGRIPTESTER.

En cuanto a la elección de tipo de pavimento se puede generalizar que los pavimentos asfalticos y de hormigón son los más usados debido a la buena correlación que existe entre calidad, coste y satisfacción en la conducción del ciclista, siempre y cuando se realice una adecuada ejecución y mantenimiento.

\section{AGRADECIMIENTOS}

Los autores agradecen a los fondos FEDER de la Unión Europea la financiación del Proyecto «Análisis de la percepcióndemanda social de los usuarios de las vías ciclistas andaluzas y estudio pre-normativo para reducir los accidentes por deslizamiento-derrape con pavimento mojado o mal tiempo (CICLOVIAS)» del «Programa Operativo FEDER de Andalucía 20072013». También agradecen a la Agencia de Obra Pública de la Consejería de Fomento y Vivienda de la Junta de Andalucía, personal e investigadores por su dedicación y profesionalidad. 


\section{REFERENCIAS}

(1) Idom. (2010). Plan Director de la bicicleta. Alhama de Murcia. http://datos.alhamademurcia.es/descargas/Plan \%20 Director \%20Bicicleta \%20de \%20Alhama \%20de \%20Murcia. \%2oDocumento \%20de \%2otrabajo.pdf.

(2) Idom. (2010). Plan Director de la bicicleta de Zaragoza. https://www.zaragoza.es/contenidos/bici/plan/CAPITULOo8.pdf._Ayuntamiento de Zaragoza.

(3) Kraemer, C., Pardillo, J.M., Rocci, S., Romana, M.G., Sánchez, V., del Val, M.A. (2004). Ingeniería de Carreteras. Volumen II. S.A. Mc Graw-Hill - Interamericana de España.

(4) Ayuntamiento de Vitoria-Gasteiz. (2010-2015). Plan Director de movilidad ciclista de Vitoria-Gasteiz, 201O-2015. Anexos. http://www.vitoria-gasteiz.org/wbo21/http/contenidosEstaticos/adjuntos/es/45/64/34564.pdf. Vitoria-Gasteizko Udala.

(5) Plan Nacional de Ciclovías. (2013). Plan maestro de Vías ciclistas de Lima y Callao (Perú). Manual de diseño para infraestructura de vías ciclistas. http://www.cicloviasecuador.gob.ec/biblioteca/doc_download/12-manual-de-disenopara-infraestructura-de-ciclovias-pdf.html.

(6) CAPA. A guideline for the design and construction of asphalt pavements for Colorado Trails and paths. http://co-asphalt.com/. Colorado (USA): Colorado Asphalt Pavement Association.

(7) California Department of Transportation. (2006). Chapter 1000. Bikeway planning and design. En Highway design manual, pp. 1-26. http://www.dot.ca.gov/hq/oppd/hdm/pdf/chp100o.pdf.

(8) ASEFMA. (2010). Monografía 11. La resistencia al deslizamiento de los pavimentos. Madrid: Asociación Española de Fabricantes de Mezclas Asfálticas.

(9) Government of South Australia. Transport Services Division. (2015). Guide to Bikeway Pavement Design Construction and Maintenance for South Australia. http://www.dpti.sa.gov.au/_data/assets/pdf_file/ooo6/149964/DPTI_Bikeway_Pavement_Guidelines_2.pdf.

(10) Ellis, R., Slate, A., Woodward, D. (2009). Micro asphalt SCRIM/griptester correlation study. En Sixth International Conference on Maintenance and Rehabilitation of Pavements and Technological Control. Politechnico di Torino, Italy. http://eprints.ulster.ac.uk/16013/1/Ellis_Slate_Woodward_Paper_39_Mairepav_6.pdf.

(11) ITDP México, A.C. e I-CE, Interface for Cycling Expertise. (2011). Ciclociudades. Manual integral de movilidad ciclista para ciudades mexicanas. Instituto para Políticas de Transporte y Desarrollo de Mexico. http://ciclociudades.mx/manual/

(12) Ayuntamiento de Málaga - Gerencia Municipal de urbanismo, obras e infraestructuras. Normas básicas de diseño para vías ciclables. https://www.ruedasredondas.org/php/adjuntos/PDB_Cap2.pdf.

(13) Ministerio de Fomento. (1999). La bicicleta en la ciudad. Manual de políticas y diseño para favorecer el uso de la bicicleta como medio de transporte. http://www.gea21.com/_media/publicaciones/la_bicicleta_en_la_ciudad_1999.pdf.

(14) Bicitekas. (2008). Criterios básicos para una infraestructura ciclista. La Rueda, $\mathrm{n}^{0}$ 2. http://bicitekas.org/wp/wp-content/uploads/2013/05/La_Rueda_2.pdf.

(15) NCDOT. (1994). Bicycle paths. En Bicycle Projects Planning and Design Guidelines. Chapter 7, pp. 41-58. North Carolina Department of Transportation. https://connect.ncdot.gov/projects/BikePed/Documents/Bicycle\%20Projects\%20 Planning\%20and\%20Design\%20Guidelines\%20-\%20Chapter\%207\%20-\%20Bicycle\%2opaths.pdf.

(16) Lai, S. J. (1975). State of the art: class I bicycle path pavements. Washington, (USA): Department of Transportation. http://www.pedbikeinfo.org/cms/downloads/StateOfArt_BikePathPavements1975.pdf.

(17) Ministerio de Fomento. Pliego de Prescripciones técnicas generales para obras de carreteras y puentes (PG-3).

(18) Consejería de Fomento y Vivienda. (2013). Recomendaciones de diseño para las vías ciclistas en Andalucía. http:// www.juntadeandalucia.es/fomentoyvivienda/estaticas/sites/consejeria/areas/transportes_infraestructuras/plan_ bici/documentos_plan_bici/recomendaciones_diseno_vias_ciclistas.pdf.

(19) Gipuzkoako Foru Aldundia. Diputación Foral de Gipuzkoa. (2006). Manual de las vías ciclistas de Gipuzkoa. Recomendaciones para su planificación y proyecto. http://www.upv.es/contenidos/CAMUNISO/info/Uo528796.pdf.

(20) Cairney, P., King, K. (2003). Development of a performance based specification for a major bicycle facility. Vermont, Victoria, Australia: ARRB Transport Research. https://www.arrb.com.au/admin/file/content13/c6/ARR358\%20developmentof\%20a\%2operformance\%20spec_1282261979669.pdf.

(21) Scottish Executive (UK) - Department for transport. (2008). Cycle infrastructure design. https://www.gov.uk/government/uploads/system/uploads/attachment_data/file/329150/ltn-2-o8_Cycle_infrastructure_design.pdf.

(22) Generalitat de Catalunya - Departament de Politica territorial i Obres Publiques. (2008). Manual para el diseño de vías ciclistas de Cataluña. http://territori.gencat.cat/web/.content/home/o1_departament/normativa_i_documentacio/ documentacio/territori_mobilitat/transport_public/publicacions/manual_per_al_disseny_de_vies_ciclistes_a_catalunya/pdf/vies_ciclistes_cast_tcm32-45417.pdf.

(23) AASHTO. (2010). Guide for the Planning, Design, and Operation of Bicycle Facilities. American Association of State Highway and Transportation Officials.

(24) Capitão, S. D., Picado-Santos, L. G., Martinho, F. (2012). Pavement engineering materials: Review on the use of warm-mix asphalt. Construction and Building Materials, 36: 1016-1024, doi: http://dx.doi.org/10.1016/j.conbuildmat.2012.06.038.

(25) Minnesota State Department of Transportation. (2007). Bikeway Facility Design Manual. http://www.dot.state.mn.us/ bike/pdfs/manual/manual.pdf.

(26) Jiménez, J. R., Ayuso, J., Agrela, F., López, M., Galvín, A.P. (2012). Utilisation of unbound recycled aggregates from selected CDW in unpaved rural roads. Resources Conservation and Recycling, 58: 88-97, doi: http://dx.doi.org/10.1016/j. resconrec.2011.10.012. 
(27) Jiménez, J.R., Ayuso, J., Galvín, A.P., López, M., Agrela, F. (2012). Use of mixed recycled aggregates with a low embodied energy from non-selected CDW in unpaved rural roads. Construction and Building Materials, 34: 34-43, doi: http://dx.doi.org/10.1016/j.conbuildmat.2012.02.042.

(28) Transport for London. (2014). Construction including surfacing. En London Cycling Design Standards, Chapter 7, pp: 280-308. http://content.tfl.gov.uk/lcds-chapter7-construction.pdf.

(29) U.S.A. Department of Transportation - Federal Highway Administration. (1992). National Bicycling and Walking Study: Current Planning Guidelines and Design Standards being used by State and Local Agencies for Bicycle and Pedestrian Facilities. http://atfiles.org/files/pdf/1992fhwadesign.PDF.

(30) City of Phoenix. (2009). Street Planning and Design Guidelines. https://www.phoenix.gov/streetssite/Documents/ d_039263.pdf. Phoenix (USA).

(31) Transportation, operations and environment division. Design guidelines for bikeways. Canada: Ontario Ministry of Transportation.

(32) CERTU. (2008). Recommandations pour les aménagements cyclables. Francia: Direction générale des Infrastructures, des Transports et de la Mer.

(33) LCN. (1998). London cycle network. Design manual. UK: Enviironmental Services, Royal Borough of Kingston. http:// www.londoncyclenetwork.org.uk/uploaded_files/LCN_Design_Manual.pdf. 